\title{
RUIN PROBABILITIES FOR TWO CLASSES OF RISK PROCESSES
}

\author{
BY \\ SHUANMING LI $^{1 *}$ AND JOSÉ GARRIDO ${ }^{2}$
}

\begin{abstract}
We consider a risk model with two independent classes of insurance risks. We assume that the two independent claim counting processes are, respectively, Poisson and Sparre Andersen processes with generalized Erlang(2) claim interarrival times. The Laplace transform of the non-ruin probability is derived from a system of integro-differential equations. Explicit results can be obtained when the initial reserve is zero and the claim severity distributions of both classes belong to the $K_{n}$ family of distributions. A relation between the ruin probability and the distribution of the supremum before ruin is identified. Finally, the Laplace transform of the non-ruin probability of a perturbed Sparre Andersen risk model with generalized Erlang(2) claim inter-arrival times is derived when the compound Poisson process converges weakly to a Wiener process.
\end{abstract}

\section{KEYWORDS}

Compound Poisson process, generalized Erlang distribution, Sparre Andersen risk model, integro-differential equations, martingales, generalized Lundberg fundamental equation, supremum distribution, Wiener process, diffusion perturbed risk model.

\section{INTRODUCTION}

Consider an insurance surplus process

$$
U(t)=u+c t-S(t), \quad t \geq 0,
$$

where $u$ is the initial surplus, $c$ is the premium rate, and $\{S(t) ; t \geq 0\}$ is the aggregate claim amount process. In this paper, we assume that $S(t)$ is generated by two classes of insurance risks. Usually such models are studied in the context of correlated risks. Our purpose is different here. We look at the impact on the

\footnotetext{
* This research was funded by a ${ }^{1} \mathrm{SOA} / \mathrm{CAS}$ Ph.D. Grant and the ${ }^{2}$ Natural Sciences and Engineering Research Council of Canada (NSERC) operating grant OGP0036860.
} 
surplus of the introduction of extra variability. Here it takes the form of the aggregate "shocks" (or claims) from the first class, independently added to the natural random variability of the aggregate insurance claims from the second class.

Actuarial models often perturb the aggregate insurance claims with diffusions. By contrast, our perturbation, in the first class, is a jump process similar to that of the insurance claims in the second class. The diffusion perturbation can still be obtained as a limit case of our model (see Section 6).

More specifically, here

$$
S(t)=S_{1}(t)+S_{2}(t)=\sum_{i=1}^{N_{1}(t)} X_{i}+\sum_{i=1}^{N_{2}(t)} Y_{i}, \quad t \geq 0
$$

where the $\left\{X_{i}\right\}_{i \geq 1}$ are the i.i.d. positive claim severities from the first class, with common distribution function $P(x)$ and density $p(x)$, while the $\left\{Y_{i}\right\}_{i \geq 1}$ are the claim severities from the second class, also assumed i.i.d. positive, but with common distribution function $Q(y)$ and density $q(y)$. Denote by $\mu_{X}$ and $\mu_{Y}$, respectively, the means of $X$ and $Y$, and by $\hat{p}(s)=\int_{0}^{\infty} e^{-s x} p(x) d x$ and $\hat{q}(s)=$ $\int_{0}^{\infty} e^{-s x} q(x) d x$ their Laplace transforms.

The claim number process $\left\{N_{1}(t) ; t \geq 0\right\}$ is assumed to be Poisson with parameter $\lambda$. The corresponding exponential claim inter-arrival times are denoted $\left\{W_{i}\right\}_{i \geq 1}$. By contrast, $\left\{N_{2}(t) ; t \geq 0\right\}$ is a renewal process with i.i.d. claim inter-arrival times $\left\{V_{i}\right\}_{i \geq 1}$ that are generalized Erlang(2) distributed, i.e. the sum $V_{i}:=L_{i 1}+L_{i 2}$ of 2 independent random variables, where the $\left\{L_{i 1}\right\}_{i \geq 1}$ are i.i.d. exponential random variables with parameter $\lambda_{1}$, while the $\left\{L_{i 2}\right\}_{i \geq 1}$ are i.i.d. exponential with parameter $\lambda_{2}$ (possibly different from $\lambda_{1}$ ).

We finally assume that $\left\{X_{i}\right\}_{i \geq 1}$ and $\left\{Y_{i}\right\}_{i \geq 1}$ are mutually independent, also independent of $N_{1}$ and $N_{2}$, and that $c>\lambda \mu_{X}+\left[\lambda_{1} \lambda_{2} /\left(\lambda_{1}+\lambda_{2}\right)\right] \mu_{Y}$, providing a positive loading factor, $\theta$, such that $1 /(1+\theta)=\left[\lambda \mu_{X}+\lambda_{1} \lambda_{2} \mu_{Y} /\left(\lambda_{1}+\lambda_{2}\right)\right] / c$.

Now define

$$
T=\inf \{t \geq 0: U(t)<0\} \quad(\infty, \text { otherwise }),
$$

to be the ruin time, and

$$
\Psi(u)=\mathbb{P}(T<\infty \mid U(0)=u), \quad u \geq 0,
$$

to be the probability of ultimate ruin (with $\Phi(u)=1-\Psi(u)$ being the survival, or non-ruin, probability).

The Erlang distribution is one of the most commonly used in queuing theory, which is closely related to risk theory. See for example, Asmussen (1987, 1989). More recently, a number of papers have discussed how to adapt the methods and results from the classical risk model to those of a Sparre Andersen model, with Erlang or generalized Erlang distributed claim inter-arrival times, see Dickson (1998), Dickson and Hipp (1998, 2001), Cheng and Tang (2003), Li (2003), Li and Garrido (2004), Gerber and Shiu (2003a,b, 2005) and the references therein. 
Yuen et al. (2002) consider the non-ruin probability for a risk process involving two dependent classes of insurance risks. It can be represented as a surplus process with two independent classes of risks, for which one claim number process is Poisson and the other is a Sparre Andersen process with Erlang(2) claim inter-arrival times. Explicit results are given only for exponentially distributed claim amounts. Their model can be reduced to the one proposed here.

We consider a risk process with two independent classes of risks, one is a compound Poisson process, the other is a compound renewal process with generalized Erlang(2) distributed claim inter-arrival times and $K_{n}$ distributed claim severities (see Willmot, 1999).

The paper is organized as follows. In Section 2, we obtain a system of integro-differential equations for the non-ruin probability $\Phi(u)$. Section 3 discusses a generalized Lundberg fundamental equation and its roots. These allow, in Section 4, to obtain and analyze the Laplace transform of the non-ruin probability. Explicit results are given when the initial reserve is zero or when the claims severity distributions for both classes belong to the $K_{n}$ family. An illustrative example with exponential severities is provided. Section 5, establishes a relationship between the ruin probability and the distribution of the supremum before ruin. Finally, in Section 6, the Laplace transform of the non-ruin probability for a Sparre Andersen risk process perturbed by a diffusion is obtained by letting the compound Poisson process converge weakly to a Brownian motion.

\section{SYSTEM OF INTEGRO-DIFFERENTIAL EQUATIONS}

In the classical risk process, the ruin probability is time homogenous due to the lack-of-memory property of the exponentially distributed claim inter-arrival times, i.e. the ruin (survival) probability, independent of time $t$, is only a function of the initial surplus. However, for our risk process, the ruin probability is no longer time-homogeneous, due to the Erlang(2) distributional assumption for the inter-arrival times from the second class. As such, for the probability of ultimate ruin $\Psi(u)$, defined in Section 1, we assume that a claim from the second class occurs exactly at time 0 .

The ruin probability, denoted by $\Psi(u, \tau)$, is a bivariate function of the current reserve $u$ and the length of time $\tau$, elapsed since the time of a claim from the second class (the surplus process repeats itself at these points). We are interested in the ruin probabilities at time zero (or at the time of a second class claim occurrence) and the time of the realization of $L_{i 1}, i=1,2, \ldots$. The former is $\Psi(u, 0)=\Psi(u)$, the latter is defined by $\Psi_{1}(u)=\mathbb{P}\left[T<\infty \mid L_{11}=t, U(t)=u\right]$, and $\Phi_{1}(u)=1-\Psi_{1}(u)$ (note that $\Psi_{1}(u)$ is independent of $t$, due to the the lack of memory of $L_{11}$ ). Then total probability formula gives

$$
\begin{aligned}
\Psi(u, \tau) & =\Psi(u) \mathbb{P}\left(L_{11}>\tau\right)+\Psi_{1}(u) \mathbb{P}\left(L_{11}<\tau\right) \\
& =e^{-\lambda_{1} \tau} \Psi(u)+\left(1-e^{-\lambda_{1} \tau}\right) \Psi_{1}(u) .
\end{aligned}
$$

Next consider the derivation of $\Psi(u)$ and $\Psi_{1}(u)$. Let $M=W_{1} \wedge \mathrm{L}_{11}$ then 


$$
\begin{aligned}
\Phi(u) & =\int_{0}^{\infty} \mathbb{P}\left(M=d t, M=L_{11}\right) \Phi_{1}(u+c t) \\
& +\int_{0}^{\infty} \mathbb{P}\left(M=d t, M=W_{1}\right) \int_{0}^{u+c t} \Phi(u+c t-x) p(x) d x .
\end{aligned}
$$

Since

$$
\begin{gathered}
\mathbb{P}\left(M=W_{1}\right)=\mathbb{P}\left(W_{1}<L_{11}\right)=\frac{\lambda}{\lambda_{1}+\lambda}, \\
\mathbb{P}\left(M=L_{11}\right)=\mathbb{P}\left(W_{1}>L_{11}\right)=\frac{\lambda_{1}}{\lambda+\lambda_{1},} \\
\mathbb{P}\left(M>t \mid M=W_{1}\right)=\mathbb{P}\left(M>t \mid M=L_{11}\right)=e^{-\left(\lambda+\lambda_{1}\right) t},
\end{gathered}
$$

then (3) can be rewritten (for $u \geq 0$ ) as:

$$
\begin{aligned}
\Phi(u)= & \frac{\lambda_{1}}{\lambda+\lambda_{1}} \int_{0}^{\infty}\left(\lambda+\lambda_{1}\right) e^{-\left(\lambda+\lambda_{1}\right) t} \Phi_{1}(u+c t) d t \\
& +\frac{\lambda}{\lambda+\lambda_{1}} \int_{0}^{\infty}\left(\lambda+\lambda_{1}\right) e^{-\left(\lambda+\lambda_{1}\right) t} \int_{0}^{u+c t} \Phi(u+c t-x) p(x) d x d t .
\end{aligned}
$$

Similarly, let $Z=W_{1} \wedge L_{12}$, then

$$
\begin{aligned}
\Phi_{1}(u) & =\int_{0}^{\infty} \mathbb{P}\left(Z=d t, Z=L_{12}\right) \int_{0}^{u+c t} \Phi(u+c t-x) q(x) d x \\
& +\int_{0}^{\infty} \mathbb{P}\left(Z=d t, M=W_{1}\right) \int_{0}^{u+c t} \Phi_{1}(u+c t-x) p(x) d x .
\end{aligned}
$$

By similar arguments, we have that (for $u \geq 0$ ):

$$
\begin{aligned}
\Phi_{1}(u)= & \frac{\lambda_{2}}{\lambda+\lambda_{2}} \int_{0}^{\infty}\left(\lambda+\lambda_{2}\right) e^{-\left(\lambda+\lambda_{2}\right) t} \int_{0}^{u+c t} \Phi(u+c t-x) q(x) d x d t \\
& +\frac{\lambda}{\lambda+\lambda_{2}} \int_{0}^{\infty}\left(\lambda+\lambda_{2}\right) e^{-\left(\lambda+\lambda_{2}\right) t} \int_{0}^{u+c t} \Phi_{1}(u+c t-x) p(x) d x d t .
\end{aligned}
$$

Setting $s=u+c t$ yields

$$
\begin{aligned}
c \Phi(u)=\lambda_{1} \int_{u}^{\infty} \Phi_{1}(s) \exp \left\{-\frac{\left(\lambda+\lambda_{1}\right)(s-u)}{c}\right\} d s \\
+\lambda \int_{u}^{\infty} \exp \left\{-\frac{\left(\lambda+\lambda_{1}\right)(s-u)}{c}\right\} \int_{0}^{s} \Phi(s-x) p(x) d x d s,
\end{aligned}
$$




$$
\begin{aligned}
c \Phi_{1}(u)=\lambda_{2} \int_{u}^{\infty} \exp \left\{-\frac{\left(\lambda+\lambda_{2}\right)(s-u)}{c}\right\} \int_{0}^{s} \Phi(s-x) q(x) d x d s \\
+\lambda \int_{u}^{\infty} \exp \left\{-\frac{\left(\lambda+\lambda_{2}\right)(s-u)}{c}\right\} \int_{0}^{s} \Phi_{1}(s-x) p(x) d x d s .
\end{aligned}
$$

Differentiating with respect to $u$ yields the following system of integro-differential equations:

$$
\begin{gathered}
c \Phi^{\prime}(u)=-\lambda_{1} \Phi_{1}(u)-\lambda \int_{0}^{u} \Phi(u-x) p(x) d x+\left(\lambda+\lambda_{1}\right) \Phi(u), \\
c \Phi_{1}^{\prime}(u)=-\lambda_{2} \int_{0}^{u} \Phi(u-x) q(x) d x-\lambda \int_{0}^{u} \Phi_{1}(u-x) p(x) d x+\left(\lambda+\lambda_{2}\right) \Phi_{1}(u) .
\end{gathered}
$$

Integrating both sides of equations (7) and (8) from 0 to $u$, after some simplifications, we obtain (for $u \geq 0$ ):

$$
\begin{gathered}
\Phi(u)=\Phi(0)+\frac{\lambda_{1}}{c} \int_{0}^{u}\left[\Phi(s)-\Phi_{1}(s)\right] d s+\frac{\lambda}{c} \int_{0}^{u} \Phi(u-x) \bar{P}(x) d x, \\
\Phi_{1}(u)=\Phi_{1}(0)+\frac{\lambda_{2}}{c} \int_{0}^{u}\left[\Phi_{1}(s)-\Phi(s)\right] d s+\frac{\lambda_{2}}{c} \int_{0}^{u} \Phi(u-x) \bar{Q}(x) d x \\
+\frac{\lambda}{c} \int_{0}^{u} \Phi_{1}(u-x) \bar{P}(x) d x
\end{gathered}
$$

where $\bar{P}(\mathrm{x})=1-P(x)$ and $\bar{Q}(x)=1-Q(x)$ are the survival functions of $P$ and $Q$, respectively. By the monotone convergence theorem and the fact that $\Phi(\infty)=$ $\Phi_{1}(\infty)=1$, it follows from (9) and (10), that as $u \rightarrow \infty$

$$
\begin{aligned}
& \Phi(0)=1-\frac{\lambda_{1}}{c} \int_{0}^{\infty}\left[\Phi(s)-\Phi_{1}(s)\right] d s+\frac{\lambda}{c} \mu_{X}, \\
& \Phi_{1}(0)=1-\frac{\lambda_{2}}{c} \int_{0}^{\infty}\left[\Phi_{1}(s)-\Phi(s)\right] d s+\frac{\lambda}{c} \mu_{X}+\frac{\lambda_{2}}{c} \mu_{Y} .
\end{aligned}
$$

Then we conclude that $\Phi(0)$ and $\Phi_{1}(0)$ satisfy the following relation:

$$
\frac{\lambda_{2}}{\lambda_{1}+\lambda_{2}} \Phi(0)+\frac{\lambda_{1}}{\lambda_{1}+\lambda_{2}} \Phi_{1}(0)=1-\frac{\lambda \mu_{X}+\frac{\lambda_{1} \lambda_{2}}{\lambda_{1}+\lambda_{2}} \mu_{Y}}{c}=\frac{\theta}{1+\theta} .
$$

Another relation between $\Phi(0)$ and $\Phi_{1}(0)$, as well as the solutions to the integrodifferential equations (7) and (8), are closely related to the root of a generalized Lundberg's equation. This is discussed in the next section. 


\section{A geNerALIZED LUNDBERG EQUATION}

Let $T_{0}=0$ and $T_{k}=\sum_{j=1}^{k} V_{j}$ be the arrival time of the $k$-th claim from the second class. Define $U_{0}=u$ and for $k=1,2, \ldots$,

$$
\begin{aligned}
U_{k}=U\left(T_{k}\right) & =u+c T_{k}-\sum_{j=1}^{k} Y_{j}-\sum_{i=1}^{N\left(T_{k}\right)} X_{i} \\
& =u+\sum_{j=1}^{k}\left[c V_{j}-Y_{j}-\sum_{i=1}^{N\left(V_{j}\right)} X_{i}\right],
\end{aligned}
$$

to be the surplus immediately after the $k$-th claim from the second class. We seek a number $s$ such that the process $\left\{e^{s U_{k}} ; k=0,1,2 \ldots\right\}$ forms a martingale. Here the martingale condition is

$$
\mathbb{E}\left[e^{c s V_{1}-s Y_{1}-s \sum_{i=1}^{N\left(Y_{1}\right)} X_{i}}\right]=\mathbb{E}\left[e^{c s V_{1}-s \sum_{i=1}^{N\left(Y_{1}\right)} X_{i}}\right] \mathbb{E}\left[e^{-s Y_{1}}\right]=1 .
$$

Since

$$
\mathbb{E}\left[e^{c s V_{1}-s \sum_{i=1}^{N\left(V_{1}\right)} X_{i}}\right]=\mathbb{E}\left[\mathbb{E}\left[e^{c s V_{1}-s \sum_{i=1}^{N\left(V_{1}\right)} X_{i}} \mid V_{1}\right]\right]=\mathbb{E}\left[e^{c s V_{1}+\lambda V_{1}\{\hat{p}(s)-1\}}\right],
$$

(12) simplifies to

$$
\mathbb{E}\left\{e^{[c s+\lambda\{\hat{p}(s)-1\}] V_{1}}\right\} \hat{q}(s)=1 .
$$

Let $\gamma(s):=\left[\frac{c}{\lambda_{1}} s+\frac{\lambda}{\lambda_{1}}(\hat{p}(s)-1)-1\right]\left[\frac{c}{\lambda_{2}} s+\frac{\lambda}{\lambda_{2}}(\hat{p}(s)-1)-1\right]$, then (13) is equivalent to

$$
\gamma(s)=\hat{q}(s), \quad s \in \mathbb{C},
$$

which is a Generalized Lundberg Fundamental Equation.

Note that $s=0$ is a root of equation (14). The following theorem shows that it also has one and only one positive real root, which plays a key role in the following sections.

Theorem 1. The generalized Lundberg equation in (14) has exactly one positive real root, say, $\rho$.

Proof. It is easy to check that the equation

$$
\gamma(s)=\left[\frac{c}{\lambda_{1}} s+\frac{\lambda}{\lambda_{1}}(\hat{p}(s)-1)-1\right]\left[\frac{c}{\lambda_{2}} s+\frac{\lambda}{\lambda_{2}}(\hat{p}(s)-1)-1\right]=0, \quad s \in \mathbb{C},
$$


has two positive real roots, say $s_{1}, s_{2}$. Noting that $c+\lambda \hat{p}^{\prime}(s) \geq c-\lambda \mu_{X}>0$, for $s \geq 0$, then

$$
\gamma^{\prime}(s)=\frac{2\left[c+\lambda \hat{p}^{\prime}(s)\right]\left[c s-\lambda-\frac{\lambda_{1}+\lambda_{2}}{2}+\lambda \hat{p}(s)\right]}{\lambda_{1} \lambda_{2}}=0,
$$

has one positive real root, say, $s_{0}$. Thus $\gamma^{\prime}(s)<0$, for $s \in\left[0, s_{0}\right)$ and $\gamma^{\prime}(s)>0$, for $s \in\left(s_{0}, \infty\right)$, hence $\gamma(s)$ is decreasing for $s \in\left[0, s_{0}\right)$, while it increases for $s \in\left(s_{0}, \infty\right)$. Furthermore, we have that $\gamma(0)=\hat{q}(0)=1$ and $\gamma^{\prime}(0)=-\frac{\left(\lambda_{1}+\lambda_{2}\right)\left(c-\lambda \mu_{X}\right)}{\lambda_{1} \lambda_{2}}<-\mu_{Y}=$ $\hat{q}^{\prime}(0)$. It follows that $\gamma(s)=\hat{q}(s)$ has only one positive real root, that we denote by $\rho$ (see Figure 1 below).

Remark: By Rouché's Theorem, the generalized Lundberg equation (14) has one and only one root on the right half complex plane, i.e., $\rho>0$ is the only root on the right half plane.

\section{LAPLACE TRANSFORMS}

In this section, we derive the Laplace transforms for the non-ruin probabilities in the integro-differential equations of Section 2, and then invert these Laplace transforms for specific claim size distributions.

Define $\hat{\Phi}(s)=\int_{0}^{\infty} e^{-s u} \Phi(u) d u$ and $\hat{\Phi}_{1}(s)=\int_{0}^{\infty} e^{-s u} \Phi_{1}(u) d u$ to be the Laplace transforms of $\Phi$ and $\Phi_{1}$, respectively. Applying a Laplace transform to both sides of equations (7) and (9) yields

$$
\left\{\begin{array}{l}
{\left[c s-\left(\lambda_{1}+\lambda\right)+\lambda \hat{p}(s)\right] \hat{\Phi}(s)=c \Phi(0)-\lambda_{1} \hat{\Phi}_{1}(s),} \\
{\left[c s-\left(\lambda_{2}+\lambda\right)+\lambda \hat{p}(s)\right] \hat{\Phi}_{1}(s)=c \Phi_{1}(0)-\lambda_{2} \hat{q}(s) \hat{\Phi}(s) .}
\end{array}\right.
$$

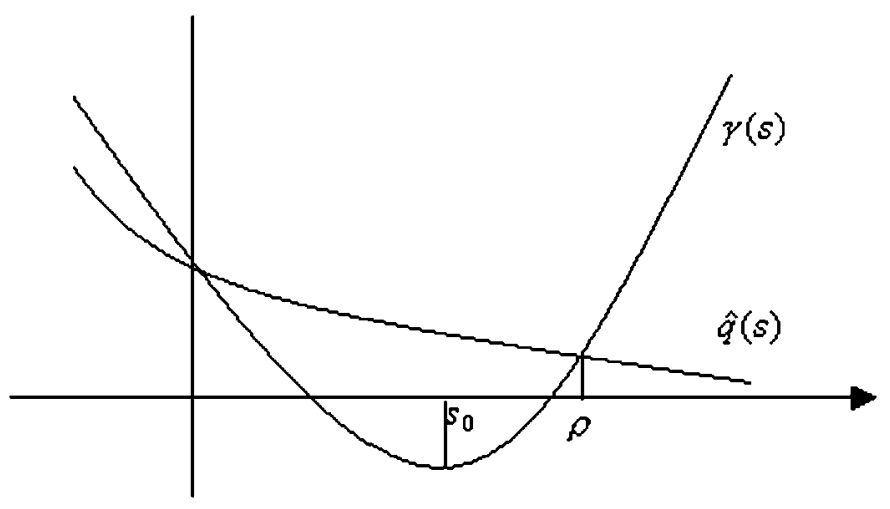

Figure 1: Roots of Generalized Lundberg Equation 
Solving the above system of equations gives

$$
\begin{gathered}
\hat{\Phi}(s)=\frac{1}{\lambda_{1} \lambda_{2}} \frac{c \Phi(0)\left[c s-\left(\lambda_{2}+\lambda\right)+\lambda \hat{p}(s)\right]-c \lambda_{1} \Phi_{1}(0)}{\gamma(s)-\hat{q}(s)}, \\
\hat{\Phi}_{1}(s)=\frac{1}{\lambda_{1} \lambda_{2}} \frac{c \Phi_{1}(0)\left[c s-\left(\lambda_{1}+\lambda\right)+\lambda \hat{p}(s)\right]-c \lambda_{2} \hat{q}(s) \Phi(0)}{\gamma(s)-\hat{q}(s)} .
\end{gathered}
$$

Since $\hat{\Phi}(s)$ and $\hat{\Phi}_{1}(s)$ are finite for all $s>0$, we have that both numerators are zero at $s=\rho$, that is

$$
\Phi(0)\left[c \rho-\left(\lambda_{2}+\lambda\right)+\lambda \hat{p}(\rho)\right]=\lambda_{1} \Phi_{1}(0) .
$$

Note that substituting $s=\rho$ in both numerators of (16) and (17) yields the same result as in (18). The approach used here is exactly the same as in Lin (2003). Therefore, equations (16) and (17) can be rewritten as

$$
\hat{\Phi}(s)=\frac{c \Phi(0)\{c(s-\rho)+\lambda[\hat{p}(s)-\hat{p}(\rho)]\}}{\lambda_{1} \lambda_{2}[\gamma(s)-\hat{q}(s)]}, \quad s \in \mathbb{C}
$$

and

$$
\begin{aligned}
\hat{\Phi}_{1}(s)= & \frac{c \Phi_{1}(0)}{c \rho-\left(\lambda_{2}+\lambda\right)+\lambda \hat{p}(\rho)} \\
& \times \frac{\left[c s-\left(\lambda_{1}+\lambda\right)+\lambda \hat{p}(s)\right]\left[c \rho-\left(\lambda_{2}+\lambda\right)+\lambda \hat{p}(\rho)\right]-\lambda_{1} \lambda_{2} \hat{q}(s)}{\lambda_{1} \lambda_{2}[\gamma(s)-\hat{q}(s)]} \\
= & \frac{c \rho-\left(\lambda_{2}+\lambda\right)+\lambda \hat{p}(\rho)}{\lambda_{1}} \hat{\Phi}(s)+\frac{c \Phi(0)}{\lambda_{1}}\left[\frac{\hat{q}(\rho)-\hat{q}(s)}{\gamma(s)-\hat{q}(s)}\right] .
\end{aligned}
$$

Solving equations (11) and (18) yields

$$
\begin{gathered}
\Phi(0)=\left(\frac{\theta}{1+\theta}\right)\left(\frac{\lambda_{1}+\lambda_{2}}{c \rho-\lambda[1-\hat{p}(\rho)]}\right) \\
\Phi_{1}(0)=\left(\frac{\theta}{1+\theta}\right)\left(\frac{\lambda_{1}+\lambda_{2}}{c \rho-\lambda[1-\hat{p}(\rho)]}\right)\left(\frac{c \rho-\lambda-\lambda_{2}+\lambda \hat{p}(\rho)}{\lambda_{1}}\right) .
\end{gathered}
$$

We now consider the case where both claims size distributions, $p$ and $q$, belong to the $K_{n}$ class, $n \in \mathbb{N}^{+}$(see Willmot, 1999), that is

$$
\hat{p}(s)=\frac{p_{n-1}(s)}{p_{n}(s)}:=\frac{\prod_{i=1}^{n} a_{i}+g(s)}{\prod_{i=1}^{n}\left(s+a_{i}\right)}, \quad n \in \mathbb{N}^{+},
$$




$$
\hat{q}(s)=\frac{q_{m-1}(s)}{q_{m}(s)}:=\frac{\prod_{i=1}^{m} b_{j}+h(s)}{\prod_{i=1}^{m}\left(s+b_{j}\right)}, \quad m \in \mathbb{N}^{+},
$$

where $a_{i}>0$, for $i=1,2, \ldots, n, b_{j}>0$, for $j=1,2, \ldots, m$ while $g(s)$ and $h(s)$ are polynomials of degree $n-1$ or less and of degree $m-1$ or less, respectively, with $h(0)=g(0)=0$. The class of $K_{n}$ distributions, widely used in applied probability applications, includes in particular the Erlang and some phase-type distributions as special cases (as well as mixture of them).

In this case, equation (19) can be transformed to a rational expression, multiplying both numerator and denominator by $\left[p_{n}(s)\right]^{2} q_{m}(s)$, that is

$$
\begin{aligned}
\hat{\Phi}(s) & =\frac{c \Phi(0)(s-\rho) p_{n}(s) q_{m}(s)\left\{c p_{n}(s)+\frac{\lambda}{p_{n}(\rho)}\left[\frac{p_{n-1}(s) p_{n}(\rho)-p_{n-1}(\rho) p_{n}(s)}{(s-\rho)}\right]\right\}}{\lambda_{1} \lambda_{2} q_{m}(s)\left[p_{n}(s)\right]^{2}[\gamma(s)-\hat{q}(s)]} \\
& =\frac{c \Phi(0)(s-\rho) p_{n}(s) q_{m}(s)\left\{c p_{n}(s)+\lambda p_{n-1}[s, \rho]-\lambda \frac{p_{n-1}(\rho)}{p_{n}(\rho)} p_{n}[s, \rho]\right\}}{\lambda_{1} \lambda_{2} q_{m}(s)\left[p_{n}(s)\right]^{2}[\gamma(s)-\hat{q}(s)]},
\end{aligned}
$$

where $p_{n-1}[s, \rho]:=\frac{p_{n-1}(s)-p_{n-1}(\rho)}{(s-\rho)}$ is the first order divided difference of $p_{n-1}(s)$ with respect to $\rho$ and $p_{n}[s, \rho]:=\frac{p_{n}(s)-p_{n}(\rho)}{(s-\rho)}$ is the first order divided difference of $p_{n}(s)$ with respect to $\rho$. Clearly, $p_{n-1}[s, \rho]$ and $p_{n}[s, \rho]$ are polynomials of degree $n-2$ and of degree $n-1$, respectively. The transform in (25) is related to the Wiener-Hopf decomposition, an approach commonly used in risk and queuing theory to identify the poles and zeros of transforms [see Cohen (1982) for details and illustrative examples]. Since our transforms take the form of ratios of polynomials, the problem here reduces to the use of partial fractions.

For simplicity, denote the denominator of (25) by $D_{2 n+m+2}(s)$. Then

$$
\begin{aligned}
D_{2 n+m+2}(s)=q_{m}(s)\left[\left(c s-\lambda_{1}-\lambda\right) p_{n}(s)+\lambda p_{n-1}(s)\right] \\
\times\left[\left(c s-\lambda-\lambda_{2}\right) p_{n}(s)+\lambda p_{n-1}(s)\right]-\lambda_{1} \lambda_{2}\left[p_{n}(s)\right]^{2} q_{m-1}(s),
\end{aligned}
$$

is a polynomial of degree $2 n+m+2$ with leading coefficient $c^{2}$. Thus the equation $D_{2 n+m+2}(s)=0$ has $2 n+m+2$ roots on the complex plane, with all the complex roots being in conjugate pairs. Noting that $s=0$ and $s=\rho$ are two of the roots, then

$$
D_{2 n+m+2}(s)=c^{2} s(s-\rho) \prod_{i=1}^{2 n+m}\left(s+R_{i}\right), \quad s \in \mathbb{C} .
$$

Note that all $R_{i}$ 's have a positive real part, since otherwise, $-R_{i}$ would also be a root of the generalized Lundberg equation (14), which is a contradiction to the conclusion that it only has one root on the right half complex plane. 
Then (25) can be simplified to

$$
\hat{\Phi}(s)=\Phi(0) \frac{p_{n}(s) q_{m}(s)\left\{p_{n}(s)+\frac{\lambda}{c} p_{n-1}[s, \rho]-\frac{\lambda p_{n-1}(\rho)}{c p_{n}(\rho)} p_{n}[s, \rho]\right\}}{s \prod_{i=1}^{2 n+m}\left(s+R_{i}\right)} .
$$

For simplicity, let $l_{2 n+m}(s)=p_{n}(s) q_{m}(s)\left\{p_{n}(s)+\frac{\lambda}{c} p_{n-1}[s, \rho]-\frac{\lambda p_{n-1}(\rho)}{c p_{n}(\rho)} p_{n}[s, \rho]\right\}$, then if $R_{1}, R_{2}, \ldots, R_{2 n+m}$ are distinct, we obtain by partial fractions that

$$
\hat{\Phi}(s)=\Phi(0)\left[\frac{c_{0}}{s}+\sum_{i=1}^{2 n+m} \frac{c_{i}}{s+R_{i}}\right] .
$$

Inverting gives

$$
\Phi(u)=\Phi(0)\left[c_{0}+\sum_{i=1}^{2 n+m} c_{i} e^{-R_{i} u}\right], \quad u>0
$$

where $c_{0}=\frac{l_{2 n+m}(0)}{\prod_{i=1}^{2 n+m} R_{i}}, c_{i}=-\frac{l_{2 n+m}\left(-R_{i}\right)}{R_{i} \prod_{j=1, j \neq i}^{n n+m}\left(R_{j}-R_{i}\right)}$, for $i=1,2, \ldots, 2 n+m$ and $\Phi(0)$ is given by (21).

From (27), we directly get that $c_{0}+\sum_{i=1}^{2 n+m} c_{i}=1$ and $c_{0}=\frac{1}{\Phi(0)}$, since $\lim _{u \rightarrow \infty}$ $\Phi(u)=1$.

Similarly, (20) can be simplified to

$$
\begin{aligned}
\hat{\Phi}_{1}(s)= & \frac{c \rho-\left(\lambda_{2}+\lambda\right)+\lambda \hat{p}(\rho)}{\lambda_{1}} \hat{\Phi}(s) \\
& +\frac{\lambda_{2} \Phi(0)}{c} \frac{\left[p_{n}(s)\right]^{2}\left\{\frac{q_{m-1}(\rho)}{q_{m}(\rho)} q_{m}[s, \rho]-q_{m-1}[s, \rho]\right\}}{s \prod_{i=1}^{2 n+m}\left(s+R_{i}\right)},
\end{aligned}
$$

where $q_{m-1}[s, \rho]=\frac{q_{m-1}(s)-q_{m-1}(\rho)}{(s-\rho)}$ and $q_{m}[s, \rho]=\frac{q_{m}(s)-q_{m}(\rho)}{s-\rho}$ are the first order divided differences of $q_{m-1}$ and $q_{m}$, respectively. For simplicity, let $h_{2 n+m-1}(s)=$ $\left[p_{n}(s)\right]^{2}\left\{\frac{q_{m-1}(\rho)}{q_{m}(\rho)} q_{m}[s, \rho]-q_{m-1}[s, \rho]\right\}$, then inverting (28) yields

$$
\Phi_{1}(u)=\frac{c \rho-\left(\lambda_{2}+\lambda\right)+\lambda \hat{p}(\rho)}{\lambda_{1}} \Phi(u)+\frac{\lambda_{2} \Phi(0)}{c}\left(d_{0}+\sum_{i=1}^{2 n+m} d_{i} e^{-R_{i} u}\right),
$$

where $d_{0}=\frac{h_{2 n+m-1}(0)}{\prod_{i=1}^{2+m} R_{i}}, d_{i}=-\frac{h_{2 n+m-1}\left(-R_{i}\right)}{R_{i} \prod_{j=1, j \neq i}^{n+m}\left(R_{j}-R_{i}\right)}$, for $i=1,2, \ldots, 2 n+m$ and $\Phi(u)$ is given by (27).

Example 1. Let $p(x)=\alpha e^{-\alpha x}, q(x)=\beta e^{-\beta x}$ be exponential distributions, with $c=(1+\theta)\left[\frac{\lambda}{\alpha}+\frac{\lambda_{1} \lambda_{2}}{\left(\lambda_{1}+\lambda_{2}\right) \beta}\right]$. Then $\hat{p}(s)=\frac{\alpha}{\alpha+s}$ and $\hat{q}(s)=\frac{\beta}{\beta+s}$, hence (21) gives 


$$
\begin{aligned}
& \Phi(0)=\frac{\theta}{1+\theta}\left[\frac{\left(\lambda_{1}+\lambda_{2}\right)(\rho+\alpha)}{c \rho^{2}+(c \alpha-\lambda) \rho}\right], \\
& \Phi_{1}(0)=\frac{\theta\left(\lambda_{1}+\lambda_{2}\right)}{(1+\theta) \lambda_{1}}\left[\frac{c \rho^{2}+\rho\left(c \alpha-\lambda-\lambda_{2}\right)-\lambda_{2} \alpha}{c \rho^{2}+(c \alpha-\lambda) \rho}\right],
\end{aligned}
$$

where $\rho$ is the positive root of the equation:

$$
\left(c s-\frac{\lambda s}{s+\alpha}-\lambda_{1}\right)\left(c s-\frac{\lambda s}{s+\alpha}-\lambda_{2}\right)=\frac{\lambda_{1} \lambda_{2} \beta}{s+\beta} .
$$

Now

$$
\begin{aligned}
D_{5}(s) & =(s+\beta)\left[c s^{2}+\left(c \alpha-\lambda-\lambda_{1}\right) s-\lambda_{1} \alpha\right]\left[c s^{2}+\left(c \alpha-\lambda-\lambda_{2}\right) s-\lambda_{2} \alpha\right] \\
& =c^{2} s(s-\rho)\left(s+R_{1}\right)\left(s+R_{2}\right)\left(s+R_{3}\right),
\end{aligned}
$$

and

$$
l_{3}(s)=(s+\alpha)(s+\beta)\left[s+\frac{c \alpha^{2}+c \rho \alpha-\lambda \alpha}{c(\rho+\alpha)}\right], \quad h_{2}(s)=\frac{\beta(s+\alpha)^{2}}{\rho+\beta}
$$

therefore

$$
\begin{gathered}
c_{0}=\frac{\alpha^{2} \beta(c \alpha+c \rho-\lambda)}{c(\rho+\alpha) R_{1} R_{2} R_{3},} c_{1}=-\frac{\left(\alpha-R_{1}\right)\left(\beta-R_{1}\right)\left[c(\rho+\alpha)\left(\alpha-R_{1}\right)-\lambda \alpha\right]}{c(\rho+\alpha) R_{1}\left(R_{2}-R_{1}\right)\left(R_{3}-R_{1}\right)}, \\
c_{2}=-\frac{\left(\alpha-R_{2}\right)\left(\beta-R_{2}\right)\left[c(\rho+\alpha)\left(\alpha-R_{2}\right)-\lambda \alpha\right]}{c(\rho+\alpha) R_{2}\left(R_{1}-R_{2}\right)\left(R_{3}-R_{2}\right)}, \\
c_{3}=-\frac{\left(\alpha-R_{3}\right)\left(\beta-R_{3}\right)\left[c(\rho+\alpha)\left(\alpha-R_{3}\right)-\lambda \alpha\right]}{c(\rho+\alpha) R_{3}\left(R_{1}-R_{3}\right)\left(R_{2}-R_{3}\right)} .
\end{gathered}
$$

Then, by (27),

$$
\begin{aligned}
\Phi(u) & =\Phi(0)\left[c_{0}+c_{1} e^{-R_{1} u}+c_{2} e^{-R_{2} u}+c_{3} e^{-R_{3} u}\right] \\
& =1+\Phi(0)\left[c_{1} e^{-R_{1} u}+c_{2} e^{-R_{2} u}+c_{3} e^{-R_{3} u}\right]
\end{aligned}
$$

Setting $c=1.5, \alpha=1, \lambda=1, \lambda_{1}=0.5, \lambda_{2}=2$ and $\beta=2$, yields $\rho=1.96372, R_{1}=$ $0.21857, R_{2}=0.79749$ and $R_{3}=1.94765$. Then $\Phi(0)=.21901, \Phi_{1}(0)=0.12395$ and

$$
\begin{gathered}
\Phi(u)=1-0.77545 e^{-0.21857 u}-0.00225 e^{-0.79749 u}-0.0032 e^{-1.94765 u} \\
\Phi_{1}(u)=1-0.85013 e^{-0.21857 u}+0.0101 e^{-0.79749 u}-0.03602 e^{-1.94765 u}
\end{gathered}
$$


Note that $\Phi(u)>\Phi_{1}(u)$ for any $u \geq 0$, as expected, due to the fact that $L_{11}+L_{12} \geq$ $L_{12}$. That is, the time to the first claim is initially longer than that at $L_{11}=t$.

\section{SuPREMUM Distribution BEFORE RUIN}

For $x>u \geq 0$, define

$$
\Gamma(u, x)=\mathbb{P}\left\{\sup _{0 \leq t \leq T} U(t) \geq x, T<\infty \mid U(0)=u\right\},
$$

to be the probability that the supremum value of the surplus process before ruin reaches or surpasses a given level $x$ when ruin occurs. Obviously $\Gamma(u, x)=\Psi(u)$, if $u \geq 0$ and $u \geq x$. As in Section 2, for $x>u \geq 0$, we define

$$
\Gamma_{1}(u, x)=\mathbb{P}\left\{\sup _{t \leq s \leq T} U(s) \geq x, T<\infty \mid L_{11}=t, U(t)=u\right\},
$$

which means that $\Gamma_{1}(u, x)=\Psi_{1}(u)=1-\Phi_{1}(u)$, if $u \geq 0$ and $u \geq x$.

We next give a system of integro-differential equations for $\Gamma(u, x)$ and $\Gamma_{1}(u, x)$.

Theorem 2. (Integro-differential equations system) $\Gamma(u, x)$ and $\Gamma_{1}(u, x)$ satisfy the following equations for $x>u \geq 0$ :

$$
\begin{gathered}
c \frac{\partial \Gamma(u, x)}{\partial u}=\left(\lambda_{1}+\lambda\right) \Gamma(u, x)-\lambda \int_{0}^{u} \Gamma(u-y, x) p(y) d y-\lambda_{1} \Gamma_{1}(u, x) \\
c \frac{\partial \Gamma_{1}(u, x)}{\partial u}=\left(\lambda_{2}+\lambda\right) \Gamma_{1}(u, x)-\lambda \int_{0}^{u} \Gamma_{1}(u-y, x) p(y) d y \\
-\lambda_{2} \int_{0}^{u} \Gamma(u-y, x) q(y) d y
\end{gathered}
$$

with boundary conditions $\Gamma(x, x)=\Psi(x)$ and $\Gamma_{1}(x, x)=\Psi_{1}(x)$.

Proof: Considering the infinitesimal interval from 0 to $d t$, letting $M=W_{1} \wedge L_{11}$ and using the law of total probability, one obtains

$$
\begin{aligned}
\Gamma(u, x) & =\mathbb{P}(M>d t) \Gamma(u+c d t, x)+\mathbb{P}\left(M<d t, M=L_{11}\right) \Gamma_{1}(u+c d t, x) \\
& +\mathbb{P}\left(M<d t, M=W_{1}\right) \int_{0}^{u+c d t} \Gamma(u+c d t-y, x) p(y) d y
\end{aligned}
$$

where

$$
\begin{aligned}
\mathbb{P}(M>d t) & =\mathbb{P}\left(W_{1}>d t\right) \mathbb{P}\left(L_{11}>d t\right) \\
& =e^{-\left(\lambda_{1}+\lambda\right) d t}=1-\left(\lambda_{1}+\lambda\right) d t+o(d t)
\end{aligned}
$$




$$
\begin{aligned}
\mathbb{P}\left(M<d t, M=L_{11}\right) & =\mathbb{P}\left(M<d t \mid L_{11}<W_{1}\right) \mathbb{P}\left(L_{11}<W_{1}\right) \\
& =\left(1-e^{-\left(\lambda_{1}+\lambda\right) d t}\right) \frac{\lambda_{1}}{\lambda_{1}+\lambda}=\lambda_{1} d t+o(d t), \\
\mathbb{P}\left(M<d t, M=W_{1}\right) & =\mathbb{P}\left(M<d t \mid L_{11}>W_{1}\right) \mathbb{P}\left(L_{11}>W_{1}\right) \\
& =\left(1-e^{-\left(\lambda_{1}+\lambda\right) d t}\right) \frac{\lambda_{1}}{\lambda_{1}+\lambda}=\lambda d t+o(d t),
\end{aligned}
$$

and

$$
\begin{aligned}
& \Gamma(u+c d t, x)=\Gamma(u, x)+c \frac{\partial \Gamma(u, x)}{\partial u} d t+o(d t), \\
& \Gamma_{1}(u+c d t, x)=\Gamma_{1}(u, x)+c \frac{\partial \Gamma_{1}(u, x)}{\partial u} d t+o(d t)
\end{aligned}
$$

Applying the above formulas to (33), subtracting $\Gamma(u, x)$ from both sides, canceling out the common factor $d t$ and letting $d t \rightarrow 0$, proves that (31) holds.

Again, considering the infinitesimal interval from $t$ to $t+d t$ and letting $Z=W_{1} \wedge L_{12}$, then by the law of total probability, one has that

$$
\begin{aligned}
\Gamma_{1}(u, x) & =\mathbb{P}(Z>d t) \Gamma_{1}(u+c d t, x) \\
& +\mathbb{P}\left(Z<d t, Z=L_{12}\right) \int_{0}^{u+c d t} \Gamma(u+c d t-y, x) q(y) d y \\
& +\mathbb{P}\left(Z<d t, Z=W_{1}\right) \int_{0}^{u+c d t} \Gamma_{1}(u+c d t-y, x) p(y) d y .
\end{aligned}
$$

Using a similar argument, gives (32) from (34).

Comparing the above integro-differential equations for $\Gamma(u, x)$ and $\Gamma_{1}(u, x)$ with (7) and (8), we have the following result.

\section{Theorem 3}

$$
\begin{gathered}
\Gamma(u, x)= \begin{cases}\frac{\Phi(u)}{\Phi(x)} \Psi(x), & \text { if } x>u \geq 0 \\
\Psi(u), & \text { if } u \geq 0, u \geq x\end{cases} \\
\Gamma_{1}(u, x)= \begin{cases}\frac{\Phi_{1}(u)}{\Phi_{1}(x)} \Psi_{1}(x), & \text { if } x>u \geq 0 \\
\Psi_{1}(u), & \text { if } u \geq 0, u \geq x\end{cases}
\end{gathered}
$$

Proof: Clearly, the conclusion holds for $u \geq 0, u \geq \mathrm{x}$. Since solutions to the integro-differential equations (31) and (32), with boundary conditions $\Gamma(x, x)=\Psi(x)$ and $\Gamma_{1}(x, x)=\Psi_{1}(x)$ are unique, noting that $\Psi(u)$ and $\Psi_{1}(u)$ satisfy equations (7) and (8), then we can conclude that for $x \geq u \geq 0, \frac{\Phi_{1}(u)}{\Phi_{1}(x)} \Psi(x)$ and $\frac{\Phi(u)}{\Phi(x)} \Psi_{1}(x)$ satisfy (31) and (32), with their respective boundary conditions. 


\section{A Sparre ANDERSEn RiSK MOdel Perturbed by DifFusion}

In this section, we obtain the ruin probability of a Sparre Andersen risk model perturbed by diffusion as a limit case, when the compound Poisson process converges weakly to a Wiener process. Although the perturbed model can be analyzed directly, the ideas here give an alternative way to analyze risk models involving Wiener processes (e.g., Gerber and Landry, 1998 and Gerber and Shiu, 1998) using only techniques for classical and Sparre Andersen models. Hereby, consider the following family of surplus processes:

$$
U_{\varepsilon}(t)=u+\left(c+c_{\varepsilon}\right) t-\varepsilon N_{1}(t)-\sum_{i=1}^{N_{2}(t)} Y_{i}, \quad \varepsilon \in \mathbb{R}^{+},
$$

where all the assumptions are as in model (1), except for the premium rate that is $c+c_{\varepsilon}$, the counting process $N_{1}$ is now a Poisson process with parameter $\lambda_{\varepsilon}$, and the claims from the first class are constant with value $\varepsilon$, while the security loading factor $\theta_{\varepsilon}$ is such that $\frac{1}{1+\theta_{\varepsilon}}=\frac{\varepsilon \lambda_{\varepsilon}+\frac{\lambda_{1} \lambda_{2}}{\lambda_{1}+\lambda_{2}} \mu_{Y}}{c+c_{\varepsilon}}$. We define

$$
T_{\varepsilon}=\inf \left\{t \geq 0: U_{\varepsilon}(t)<0 \mid U_{\varepsilon}(0)=u\right\},
$$

to be the ruin time corresponding to risk model (37), while

$$
\Psi_{\varepsilon}(u)=\mathbb{P}\left(T_{\varepsilon}<\infty\right), \quad u \geq 0,
$$

is the ruin probability, $\Phi_{\varepsilon}(u)=1-\Psi_{\varepsilon}(u)$ the corresponding survival probability, and $\hat{\Phi}_{\varepsilon}(s)=\int_{0}^{\infty} e^{-s u} \Phi_{\varepsilon}(u) d u$ the Laplace transform of $\Phi_{\varepsilon}$. Then

$$
\Phi_{\varepsilon}(0)=\frac{\theta_{\varepsilon}}{1+\theta_{\varepsilon}}\left[\frac{\lambda_{1}+\lambda_{2}}{\left(c+c_{\varepsilon}\right) \rho_{\varepsilon}-\lambda_{\varepsilon}\left(1-e^{-\varepsilon \rho_{\varepsilon}}\right)}\right]
$$

and

$$
\hat{\Phi}_{\varepsilon}(s)=\frac{\left(c+c_{\varepsilon}\right) \Phi_{\varepsilon}(0)\left[\left(c+c_{\varepsilon}\right)\left(s-\rho_{\varepsilon}\right)+\lambda_{\varepsilon}\left(e^{-s \varepsilon}-e^{-\rho_{\varepsilon} \varepsilon}\right)\right]}{\lambda_{1} \lambda_{2}\left[\gamma_{\varepsilon}(s)-\hat{q}(s)\right]},
$$

where $\gamma_{\varepsilon}(s)=\left[\frac{c+c_{\varepsilon}}{\lambda_{1}} S+\frac{\lambda_{\varepsilon}}{\lambda_{1}}\left(e^{-s \varepsilon}-1\right)-1\right]\left[\frac{c+c_{\varepsilon}}{\lambda_{2}} s+\frac{\lambda_{\varepsilon}}{\lambda_{2}}\left(e^{-s \varepsilon}-1\right)-1\right]$, and $\rho_{\varepsilon}$ is the unique positive real root of the equation

$$
\gamma_{\varepsilon}(s)=\hat{q}(s), \quad s \in \mathbb{C} .
$$

Now, for a fixed constant $\sigma$, we choose $\varepsilon, \lambda_{\varepsilon}$ and $c_{\varepsilon}$ such that

$$
\mathbb{E}\left[c_{\varepsilon} t-\varepsilon N_{1}(t)\right]=0 \quad \text { and } \mathbb{V}\left[\varepsilon N_{1}(t)\right]=\sigma^{2} t .
$$


The two conditions above give that $\lambda_{\varepsilon}=\frac{\sigma^{2}}{\varepsilon^{2}}$ and $c_{\varepsilon}=\frac{\sigma^{2}}{\varepsilon}$. Then the cumulant generating function of the shifted compound Poisson process $c_{\varepsilon} t-\varepsilon N_{1}(t)$ is given by

$$
\begin{aligned}
\ln \mathbb{E}\left[e^{z\left(c_{\varepsilon} t-\varepsilon N_{1}(t)\right)}\right] & =c_{\varepsilon} z t+\lambda_{\varepsilon} t\left(e^{-\varepsilon z}-1\right)=\frac{\sigma^{2} z t}{\varepsilon}+\frac{\sigma^{2} t\left(e^{-z \varepsilon}-1\right)}{\varepsilon^{2}} \\
& =\frac{\sigma^{2}}{2} z^{2} t-\frac{z^{3} t}{3 !} \varepsilon+\frac{z^{4} t}{4 !} \varepsilon^{2}+\cdots .
\end{aligned}
$$

If $\varepsilon \rightarrow 0^{+}$, then $\mathbb{E}\left[e^{z\left(c_{\varepsilon} t-\varepsilon N_{1}(t)\right)}\right] \rightarrow e^{\frac{\sigma^{2} z^{2}}{2}}=\mathbb{E}\left[e^{z \sigma B(t)}\right]$, where $\{B(t) ; t \geq 0\}$ is a standard Wiener process. This shows that the process $\left\{c_{\varepsilon} t-\varepsilon N_{1}(t) ; t \geq 0\right\}$ converges weakly to the process $\{\sigma B(t) ; t \geq 0\}$, therefore the surplus process $U_{\varepsilon}(t)$ defined in (37) converges weakly to the process:

$$
U_{0}(t)=u+c t-\sum_{i=1}^{N_{2}(t)} Y_{i}+\sigma B(t), \quad t \geq 0 .
$$

(41) is a Sparre Andersen model perturbed by a diffusion, in which the claim inter-arrival times are generalized Erlang(2) distributed.

Furthermore, when $\varepsilon \rightarrow 0^{+}$

$$
\begin{aligned}
\gamma_{\varepsilon}(s) & =\left[\frac{c+c_{\varepsilon}}{\lambda_{1}} s+\frac{\lambda_{\varepsilon}}{\lambda_{1}}\left(e^{-s \varepsilon}-1\right)-1\right]\left[\frac{c+c_{\varepsilon}}{\lambda_{2}} s+\frac{\lambda_{\varepsilon}}{\lambda_{2}}\left(e^{-s \varepsilon}-1\right)-1\right] \\
& \rightarrow \gamma_{0}(s):=\frac{1}{\lambda_{1} \lambda_{2}}\left[\lambda_{1}-c s-\frac{\sigma^{2}}{2} s^{2}\right]\left[\lambda_{2}-c s-\frac{\sigma^{2}}{2} s^{2}\right],
\end{aligned}
$$

$\rho_{\varepsilon} \rightarrow \rho_{0}$, where $\rho_{0}$ is the unique positive real root of the equation $\gamma_{0}(s)=\hat{q}(s)$, $\frac{\theta_{\varepsilon}}{1+\theta_{\varepsilon}}=\frac{c-\frac{\lambda_{1} \lambda_{2}}{\lambda_{1}+\lambda_{2}} \mu_{Y}}{\frac{\sigma^{2}}{\varepsilon}+c} \rightarrow 0$ and

$$
\begin{aligned}
\Phi_{\varepsilon}(0) & =\frac{\theta_{\varepsilon}}{1+\theta_{\varepsilon}}\left[\frac{\lambda_{1}+\lambda_{2}}{\left(c+c_{\varepsilon}\right) \rho_{\varepsilon}-\lambda_{\varepsilon}\left(1-e^{-\varepsilon \rho_{\varepsilon}}\right)}\right] \rightarrow 0 \times \frac{\lambda_{1}+\lambda_{2}}{c \rho_{0}+\frac{\sigma^{2} \rho_{0}^{2}}{2}}=0 \\
\left(c+c_{\varepsilon}\right) \Phi_{\varepsilon}(0) & =\left(c+c_{\varepsilon}\right)\left(\frac{\theta_{\varepsilon}}{1+\theta_{\varepsilon}}\right)\left[\frac{\lambda_{1}+\lambda_{2}}{\left(c+c_{\varepsilon}\right) \rho_{\varepsilon}-\lambda_{\varepsilon}\left(1-e^{-\varepsilon \rho_{\varepsilon}}\right)}\right] \\
& =\left(c-\frac{\lambda_{1} \lambda_{2}}{\lambda_{1}+\lambda_{2}} \mu_{Y}\right)\left[\frac{\lambda_{1}+\lambda_{2}}{\left(c+c_{\varepsilon}\right) \rho_{\varepsilon}-\lambda_{\varepsilon}\left(1-e^{-\varepsilon \rho_{\varepsilon}}\right)}\right] \\
& \rightarrow\left(c-\frac{\lambda_{1} \lambda_{2}}{\lambda_{1}+\lambda_{2}} \mu_{Y}\right)\left(\frac{\lambda_{1}+\lambda_{2}}{c \rho_{0}+\frac{\sigma^{2} \rho_{0}^{2}}{2}}\right) .
\end{aligned}
$$


Finally, taking the limit of (39) when $\varepsilon \rightarrow 0^{+}$gives,

$$
\hat{\Phi}_{\varepsilon}(s) \rightarrow \hat{\Phi}_{0}(s):=\left[\frac{c\left(\lambda_{1}+\lambda_{2}\right)-\lambda_{1} \lambda_{2} \mu_{Y}}{c \rho_{0}+\frac{\sigma^{2} \rho_{0}^{2}}{2}}\right] \frac{\left(s-\rho_{0}\right)\left(\frac{\sigma^{2}}{2} s+\frac{\sigma^{2} \rho_{0}}{2}+c\right)}{\lambda_{1} \lambda_{2}\left[\gamma_{0}(s)-\hat{q}(s)\right]} .
$$

The limit in (42) gives the Laplace transform of the survival probability $\Phi_{0}(u)$ for the perturbed risk model (41). In particular, if $\sigma=0$, then (42) simplifies to

$$
\hat{\Phi}_{0}(s)=\left[\frac{c\left(\lambda_{1}+\lambda_{2}\right)-\lambda_{1} \lambda_{2} \mu_{Y}}{s_{0}}\right] \frac{\left(s-s_{0}\right)}{\left[\left(\lambda_{1}-c s\right)\left(\lambda_{2}-c s\right)-\lambda_{1} \lambda_{2} \hat{q}(s)\right]},
$$

where $s_{0}$ is the unique positive solution of the equation $\left(\lambda_{1}-c s\right)\left(\lambda_{2}-c s\right)-$ $\lambda_{1} \lambda_{2} \hat{q}(s)=0$. Furthermore, if $\lambda_{1}=\lambda_{2}=\beta$, then (43) simplifies to

$$
\hat{\Phi}_{0}(s)=\frac{\frac{2 c \beta-\beta^{2} \mu_{Y}}{s_{0}} s+\beta^{2} \mu_{Y}-2 c \beta}{c^{2} s^{2}-2 c \beta s+\beta^{2}[1-\hat{q}(s)]} \text {. }
$$

We note that (44) is the Laplace transform of the non-ruin probability for the Erlang(2) risk process, and can be found in Dickson and Hipp (1998).

\section{CONCLUDING REMARKS}

In this paper, we show how to calculate the ruin (or survival) probability for a risk process with two independent classes of business, one is from the classical risk process, the other is from a generalized Erlang(2) risk process.

The results can be extended in various directions, for example, ruin probabilities can be decomposed as the ruin probability caused by a claim from the first class, or the ruin probability caused by a claim from the second class. The model also can be extended to a risk process with two dependent classes, or two independent classes, one being compound Poisson and the other generalized Erlang $(n)$.

\section{REFERENCES}

Asmussen, S. (1987) Applied Probability and Queues. Wiley, New York.

Asmussen, S. (1989) Risk theory in a Markovian environment. Scandinavian Actuarial Journal, $69-100$.

Cheng, Y. and TAng, Q. (2003) Moments of the surplus before ruin and the deficit at ruin in the Erlang(2) risk process. North American Actuarial Journal, 7(1), 1-12.

Cohen, J.W. (1982) The Single Server Queue, Rev. Ed., North-Holland, Amsterdam.

Dickson, D.C.M. (1998) On a class of renewal risk process. North American Actuarial Journal, 2(3), 60-68.

Dickson, D.C.M. and HipP, C. (1998) Ruin probabilities for Erlang(2) risk processes. Insurance: Mathematics and Economics, 22, 251-262. 
Dickson, D.C.M. and Hipp, C. (2001) On the time to ruin for Erlang(2) risk processes. Insurance: Mathematics and Economics, 29, 333-344.

Gerber, H.U. and LANDRY, B. (1998) On the discounted penalty at ruin in a jump-diffusion and the perpetual put option. Insurance: Mathematics and Economics, 22, 263-276.

Gerber, H.U. and ShiU, E.S.W. (1998) Pricing perpetual option for jump processes. North American Actuarial Journal, 2(3), 101-107.

Gerber, H.U. and Shiu, E.S.W. (2003a) Discussion of Yebin Cheng and Qihe Tang's "Moments of the surplus before ruin and the deficit at ruin". North American Actuarial Journal, 7(3), $117-119$.

Gerber, H.U. and Shiu, E.S.W. (2003b) Discussion of Yebin Cheng and Qihe Tang's "Moments of the surplus before ruin and the deficit at ruin". North American Actuarial Journal, 7(4), 96-101.

Gerber, H.U. and ShiU, E.S.W. (2005) On the time value of ruin in a Sparre Andersen risk process. North American Actuarial Journal, 9(2), in press.

LI, S. (2003) Discussion of Yebin Cheng and Qihe Tang's "Moments of the surplus before ruin and the deficit at ruin". North American Actuarial Journal, 7(3), 119-122.

LI, S. and GARRIDO, J. (2004) On ruin for the $\operatorname{Erlang}(n)$ risk process. Insurance: Mathematics and Economics, 34(3), 391-408.

LIN, X.S. (2003) Discussion of Yebin Cheng and Qihe Tang's "Moments of the surplus before ruin and the deficit at ruin". North American Actuarial Journal, 7(3), 122-124.

WiLLmot, G.E. (1999) A Laplace transform representation in a class of renewal queuing and risk process. Journal of Applied Probability, 36, 570-584.

YuEN, K.C., GuO, J. and Wu, X. (2002) On a correlated aggregate claims model with Poisson and Erlang risk processes. Insurance: Mathematics and Economics, 31, 205-214.

SHUANMING LI

Centre for Actuarial Studies

Faculty of Economics and Commerce

University of Melbourne

Parkville 3052

Victoria, Australia

Email:shli@unimelb.edu.au

JoSÉ GARRIDO

Department of Mathematics and Statistics

Concordia University

7141 Sherbrooke Street West

Montréal, Québec

H4B 1R7 Canada

Email: garrido@mathstat.concordia.ca 